\title{
Molecular cloning and expression of the porcine $S 14 R$ gene in Escherichia coli
}

\author{
Y.J. Guo ${ }^{1 *}$, G.Z. Liu ${ }^{1 *}$, C.M. Wang ${ }^{1}$, Y.Y. Wang ${ }^{2}$, H.J. Li ${ }^{2}$, K. Zhong ${ }^{2}$, \\ W.F. Lu" ${ }^{2}$, Y.L. Wang ${ }^{2}$, G.Y. Yang ${ }^{2}$ \\ ${ }^{1}$ College of Animal Sciences and Veterinary Medicine, \\ Henan Agricultural University, Zhengzhou, Henan Province, China \\ ${ }^{2}$ Key Laboratory of Animal Biochemistry and Nutrition, Henan Agricultural University, \\ Ministry of Agriculture, Zhengzhou, Henan Province, China \\ *These authors contributed equally to this study. \\ Corresponding authors: Y. L. Wang / G. Y. Yang \\ E-mail: cngyuj@163.com
}

Genet. Mol. Res. 12 (4): 4405-4412 (2013)

Received September 5, 2012

Accepted May 12, 2013

Published October 10, 2013

DOI http://dx.doi.org/10.4238/2013.October.10.6

\begin{abstract}
We amplified S14R protein gene cDNA of porcine, cloned it into a prokaryotic expression plasmid, and expressed it in Escherichia coli. A pair of primers was designed based on the cDNA sequence of the porcine $S 14 R$ gene in GenBank. The target gene fragment from porcine liver tissue was amplified by RT-PCR. Confirmed by auto-sequencing, the target gene fragment was subcloned into an expression vector of pET28a. The pET28a-S14R construct was subsequently transformed into $E$. coli BL21 (DE3). This construct was verified by restriction endonuclease digestion and sequencing. Using isopropyl $\beta$-D-1-thiogalactopyranoside induction, a new recombinant protein with the expected relative molecular mass of $24 \mathrm{kDa}$ appeared. The result was identified by SDS-PAGE electrophoresis. Porcine S14R includes 549bp (GenBank No. JN793537), with an open reading frame of 549 bp coding 182 amino acids.
\end{abstract}

Key words: MIDIIP1; Gene cloning; Prokaryotic expression; Porcine 


\section{INTRODUCTION}

The thyroid hormone-responsive spot14 (THRSP) (or Spot14, S14) responds to thyroid hormone stimulation (Seelig et al., 1981; Liaw and Towle, 1984; Jump et al., 1984). S14 is a small acidic nuclear protein that is a transcription factor involved in the transcriptional control of lipogenic enzymes (Brown et al., 1997). Some publications have reported that S14 plays important roles in the regulation of lipogenesis (Kinlaw et al., 1995; Zhu et al., 2001, 2005). The S14-related gene (S14R) is a paralog of S14 (Tsatsos et al., 2008). In 2004, using a yeast two-hybrid screening approach, Berti et al. identified the $S 14 R$ gene, whose protein product interacted with midlinel (Mid1) to stabilize microtubules during embryonic development. S14R has therefore also been called Mid1-interacting G12-like protein (MIG12); however, its official name is now Mid1 interacting protein (MIDIIP1). MIDIIP1 is located on the X chromosome (Zhu et al., 2005), but S14 on autosomal chromosomes (Grillasca et al., 1997). MID1IP1 is a cytoplasmic protein (Kim et al., 2010), while S14 is a nuclear protein. In addition, $S 14$ is predominately expressed in liver, adipose tissues and lactating mammary gland (Jump and Oppenheimer, 1985; Freake and Oppenheimer, 1987), while MIDIIP1 is widely expressed in multiple tissues but not in mammary gland. Just like with $S 14$ function, recent research (Aipoalani et al., 2010) has demonstrated that MIDIIP1 has essential roles in the regulation of lipogenesis.

Lipid metabolism is very important for the improvement of meat quality. Pork is an important food, and its quality directly correlates with human health concerns, such as obesity and cardiovascular disease. As an important factor in regulating lipid metabolism, MIDIIPI has previously been studied, but the function of porcine MIDIIPI requires further investigation. In this study, we extracted total RNA from porcine liver and cloned an MIDIIP1 cDNA by RT-PCR. We then cloned MIDIIP1 into a prokaryotic expression vector, pET28a, and expressed MIDIIP1 in Escherichia coli at high levels. This provides a basis for studying the function of porcine MIDIIPI.

\section{MATERIAL AND METHODS}

\section{Animal and sample collection}

Four pigs (averaging $45 \mathrm{~kg}$ ) of the landrace were collected from Yongkang pig breeding farm in Kaifeng of Henan Province. Liver tissue obtained from pigs was immediately dissected, frozen in liquid nitrogen, and stored at $-80^{\circ} \mathrm{C}$.

\section{Total RNA isolation and cDNA synthesis}

Total RNA was isolated from frozen porcine liver tissue using Trizol (Gibco-BRL, Germany) according to the manufacturer instructions. All extracted RNA samples were dissolved in RNase-free water. The purity and quantity of total RNA were measured with an ultraviolet/visible spectrophotometer (Nanodrop 2000/2000C, USA). First strand cDNA was synthesized using MMLV reverse transcriptase (Promega, Madison, WI, USA), $3 \mu \mathrm{g}$ RNA, and an oligo $(\mathrm{dT})_{18}$ primer. 


\section{Amplification of the MID1IP1 gene by RT-PCR}

The primers were designed on the basis of the human and rat MIDIIP1 mRNA (NM_001098791.1 and NM_206950.1) published in GenBank: forward primer (containing EcoRI restriction enzyme site), 5'-CGGAATTCATGATGCAAATTTGCGACAC-3', and reverse primer (containing HindIII restriction enzyme site), 5'-CCCAAGCTTTCAGTGCCC CCAATTGCTG-3'. RT-PCR was performed using the cDNA above. The $25-\mu \mathrm{L}$ reaction solution contained $1.0 \mu \mathrm{L}$ cDNA, $1.0 \mu \mathrm{L} 20 \mathrm{pM}$ forward primer, $1.0 \mu \mathrm{L} 20 \mathrm{pM}$ reverse primer, $12.5 \mu \mathrm{L} 2 \mathrm{X}$ Master mix (Shanghai Wonhon Biotechnology Company), and $9.5 \mu \mathrm{L}$ sterile water. The PCR reaction was started with pre-denaturation at $94^{\circ} \mathrm{C}$ for 4 min, followed by 30 cycles of denaturation at $94^{\circ} \mathrm{C}$ for $40 \mathrm{~s}$, annealing at $58^{\circ} \mathrm{C}$ for $40 \mathrm{~s}$ and extension at $72^{\circ} \mathrm{C}$ for $50 \mathrm{~s}$, completed with a final extension at $72^{\circ} \mathrm{C}$ for $10 \mathrm{~min}$. Ten microliters of PCR reaction were electrophoresed on a $1.0 \%$ agarose gel. The DNA band of the expected size was purified using a QIAquick Gel Extraction kit (Qiagen, Germany), ligated into pMD19-T simple vector (Takara, Dalian, China), and then sequenced (Sangon, Shanghai, China). The resulting sequences were deposited in GenBank and submitted to the BLAST program.

\section{Sequence analysis}

The molecular weight (MW) and the theoretical isoelectric point (pI) were calculated by ExPaSy (http://expasy.ch/tools/protparam.html). Sequence analysis of porcine MIDIIPI was carried out using the BLAST program at the National Center for Biotechnology Information (NCBI) server (http://www.nibi.nlm.nih.gov/BLAST). The prediction of signal peptides was carried out using SignalP 3.0 (http://www.cbs.dtu.dk/services/Signalp). The protein secondary structure was predicted by SABLE (http://sable.cchmc.org).

\section{Construction of a recombinant MID1IP1 expression vector}

Both pMD19-T-MIDIIP1 and pET28a (Takara) plasmids were digested with HindIII and EcoRI endonucleases. The fragments of MIDIIP1 and pET28a were gel-purified (Qiagen), and were then linked in the presence of T4 DNA ligase (Takara, Dalian, China). The reaction system contained $2 \mu \mathrm{L}$ buffer, $10 \mu \mathrm{L}$ MIDIIP1 fragments, $5 \mu \mathrm{L}$ pET28a vector fragments, $1 \mu \mathrm{L}$ T4 DNA ligase and $2 \mu \mathrm{L}$ ultrapure water. After $12 \mathrm{~h}$ at $16^{\circ} \mathrm{C}$, the recombinant plasmid pET28MID1IP1 was transformed into E. coli BL21 (DE3). The pET28-MIDIIP1 recombinant plasmid was confirmed by colony PCR and double digestion with HindIII and EcoRI.

\section{Protein expression in E. coli BL21 (DE3)}

Single colonies of pET28-MID1IP1 in E. coli BL21 (DE3) were added to LB medium containing $50 \mu \mathrm{g} / \mathrm{mL}$ ampicillin and cultured at $37^{\circ} \mathrm{C}$ overnight. Fresh LB was then inoculated 1:100 with the overnight culture and cells grown until an $\mathrm{OD}_{600}$ of 0.4-0.6. IPTG at a final concentration of $1 \mathrm{mM}$ was then added to induce expression, and cultures were incubated for another $8 \mathrm{~h}$. Cells were then pelleted at 12,000 rpm for $1 \mathrm{~min}$ and $100 \mu \mathrm{L}$ SDS loading buffer per $1.5 \mathrm{ml}$ cells added. SDS-PAGE was carried out using the Bio-Rad Mini-protein system (USA). A $12 \%$ SDS-PAGE was used to analyze the recombinant protein. The protein bands were stained with Coomassie Brilliant Blue R-250 for visualization, after electrophoresis. 


\section{RESULTS}

\section{Molecular cloning of MID1IP1}

The MIDIIP1 RT-PCR products were analyzed by agarose gel electrophoresis. A fragment of the expected size, $\sim 549 \mathrm{bp}$, was detected (Figure 1), isolated, purified and cloned into pMD19-T. After transformation and overnight culture, purified pMD19-T-MID1IP1 plasmids were analyzed by PCR (Figure 2).

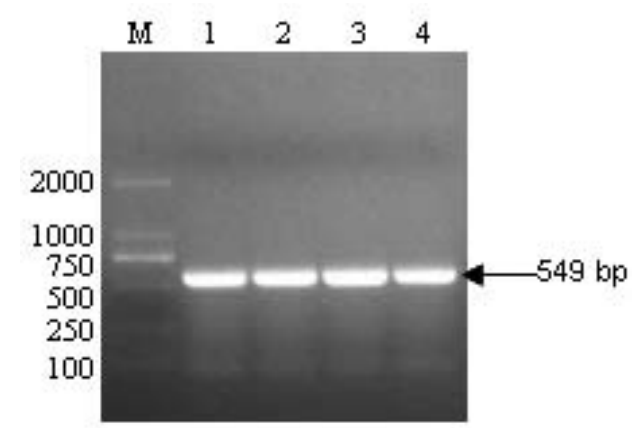

Figure 1. Gel electrophoresis of RT-PCR products. Lane $M=$ Marker DL2000; lanes 1-4 = expected PCR product of $\sim 549 \mathrm{bp}$.

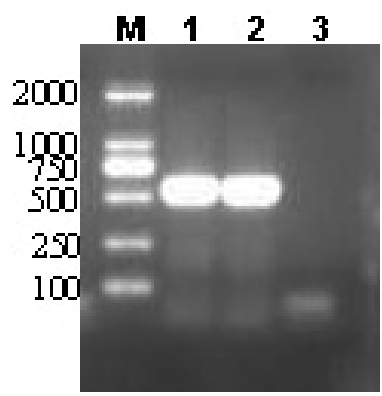

Figure 2. PCR identification of pMD19-T-S14R by gene-specific (GS) primers of S14R. Lane M= Marker DL2000; lanes 1-3 = PCR of pMD19-T-S14R by GS primers of $S 14 R$.

\section{Sequence analysis}

Porcine MIDIIP1 showed an open reading frame of $549 \mathrm{bp}$, encoding 182 coding amino acids. From the deduced amino acid sequence, the MW and pI of porcine MID1IP1 are 20186.6 and 5.51, respectively. The cDNA nucleotide sequence analysis running the BLAST program showed that the porcine MIDIIPI had high homology with the human (95\%), mouse (90\%), rat $(90 \%)$, and cattle (95\%) protein. It was submitted to the GenBank database (JN793537). The prediction of signal peptide by SignalP indicated that the porcine MID1IP1 protein had no signal peptide. SABLE on-line analysis software was applied and full-automated mode was selected to conduct predictive parsing for protein sequence secondary structure. The porcine MID1IP1 protein contained three alpha-helix, one $\beta$-sheet and random coils (Figure 3). 


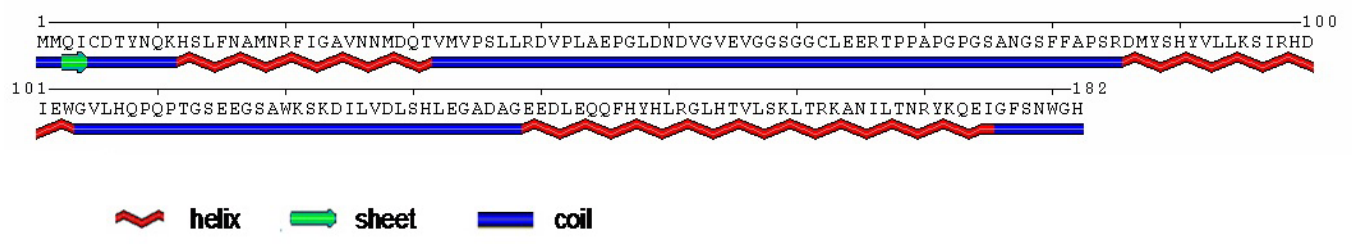

Figure 3. The secondary structure of porcine S14R protein was constructed by SABLE.

\section{Construction of recombinant MID1IP1 expression vector}

After digestion of pMD19-T-MID1IP1 and pET28a (Takara) with HindIII and EcoRI endonucleases, the resulting DNA fragments were gel-purified. A 549-bp restriction fragment from the sequenced pMD19-T-MIDIIP1 plasmid was subcloned into the HindIII and EcoRI sites of pET28a (Takara) in frame with the N-terminal polyhistidine (6xHis) tag. The recombinant plasmid pET28-MID1IP1 was then transformed into E. coli BL21 (DE3). After transformation and overnight culture, the pET28-MIDIIP1 recombinant plasmid was isolated and confirmed by colony PCR (Figure 4) and restriction enzyme digestion analysis (Figure 5). Sequence analysis of pET28-MIDIIP1 showed that the insert orientation and position of MIDIIP1 were correct, which indicated that the cloned porcine MIDIIP1 gene could be induced to express protein.

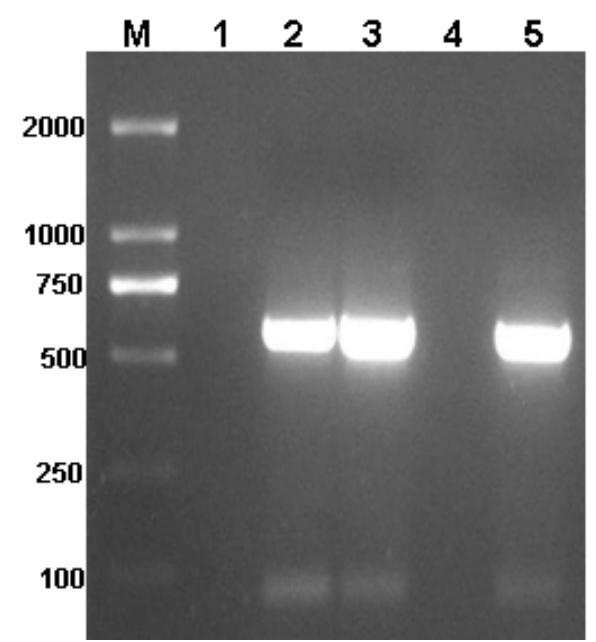

Figure 4. PCR identification of pET28-S14R by gene-specific (GS) primers of S14R. Lane M= Marker DL2000; lanes $1-5=$ PCR of pET28-S14R by GS primers of S14R.

\section{MID1IP1 gene expressed in $E$. coli}

The pET28-MID1IP1 was transformed into E. coli BL21 (DE3) and was induced with IPTG to express MID1IP1. The cell lysates were separated by $12 \%$ SDS-PAGE electrophoresis, which showed that a protein with a molecular weight of about $24 \mathrm{kD}$ was expressed, with no such band in the non-IPTG-induced control sample, indicating that the $24-\mathrm{kD}$ protein band was the expression product of MID1IP1 (Figure 6). 


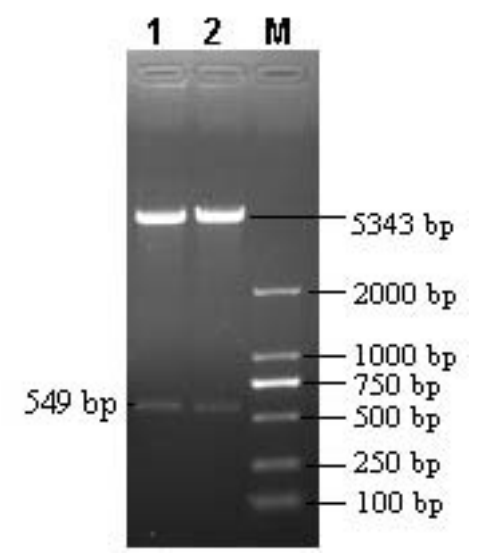

Figure 5. HindIII/ EcoRI digestion analysis of pET28-S14R recombinants. Lane $M=$ Marker DL2000; lanes 1 and $2=$ pET28-S14R candidates digested by HindIII/EcoRI.

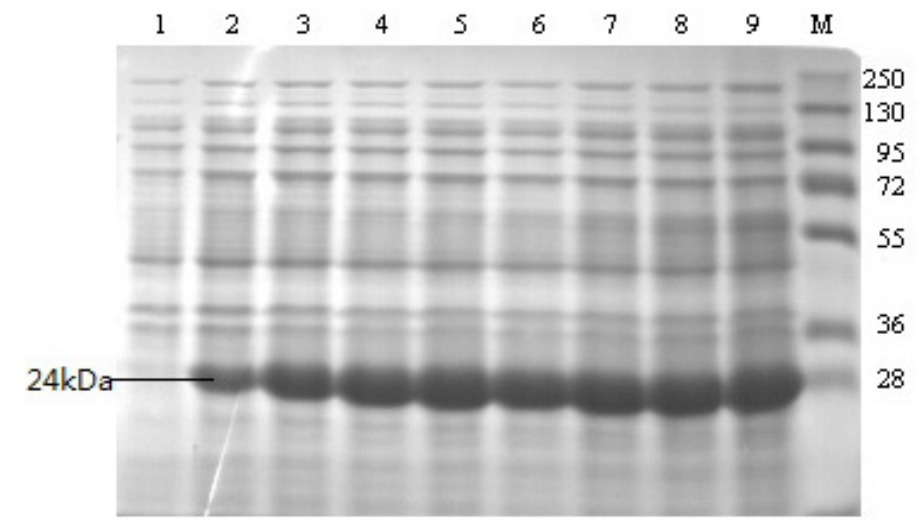

Figure 6. SDS-PAGE analysis of pET28-MIDIIP1 expression in Escherichia coli BL21. Lane 1= BL21/pET28MIDIIPI without IPTG induction; lanes $2-9$ = induced with IPTG for 1, 2, 3, 4, 5, 6, 7, 8 h respectively. Arrow indicates the expressed MID1IP1 fusion protein; lane $M=$ protein marker

\section{DISCUSSION}

Although MID1IP1 shared a low amino acid homology with S14, as the paralog of S14, some studies (Kim et al., 2010; Colbert et al., 2010) have reported that it plays important roles in lipid metabolism. As we all know, acetyl-coenzyme A carboxylase (ACC) is a ratelimiting enzyme for de novo fatty acid synthesis. Recent studies have shown that MID1IP1 is an ACC-binding protein (Kim et al., 2010). MID1IP1 can activate acetyl-coenzyme A carboxylase. Another recent report has revealed that MID1IP1 is a new liver X receptor (LXR) target (Inoue et al., 2011). In this paper, the open reading frame (ORF) of porcine MIDIIP1 was isolated. It contains 549 nucleotides encoding 182 amino acids. These data will provide the molecular basis for porcine MIDIIP1 analysis of nucleotide sequence and deduced amino acid sequence. BLAST analysis of the deduced amino acid sequence demonstrated that porcine MID1IP1 had high homology with the human (95\%), mouse (90\%), rat (90\%), and bovine 
(95\%) molecule. Signal peptide analysis showed that the MID1IP1 protein is a non-secretory protein. MID1IP1 is a cytoplasmic protein, which has been confirmed in humans and mice (Berit et al., 2004; Kim et al., 2010). To further investigate the detailed structure of porcine MID1IP1, SABLE on-line analysis software was employed to determine secondary structure. Our results indicate that the secondary structure of porcine MID1IP1 protein was the mixed type, including alpha-helix, $\beta$-sheet and random coils.

To study the function of porcine MID1IP1 and S14, we had previously completed the cloning, expression and affinity purification of porcine S14 (Wang et al., 2012). On this basis, we first obtained the full length coding sequence of porcine MIDIIP1. Prokaryotic expression can produce large quantities of recombinant proteins with biological activity, which can be used for further functional studies. After EcoRI and HindIII digestion, the full length gene encoding porcine MIDIIP1 was inserted into prokaryotic expression plasmid pET28a. The recombination plasmid pET28-MIDIIP1 was transformed into E.coli BL21 (DE3), and expressed upon IPTG induction. Different expression conditions were explored and a 4-h period of IPTG induction at a final concentration of $1 \mathrm{mM}$ was found to be optimal for high level expression of the MID1IP1-His ${ }_{6}$ fusion protein, with a molecular weight of about $24 \mathrm{kD}$. The pET28a prokaryotic expression vector incorporates a $6 \times$ His tag. This enables large quantities of the recombinant MID1IP1-His ${ }_{6}$ fusion protein to be purified by the standard procedure of nickel-based affinity chromatography.

To our knowledge, this study is the first to report the cloning and expression in $E$. coli of porcine MID1IP1. The recombinant prokaryotic expression vector pET28-MID1IP1 was constructed, and MID1IP1-His ${ }_{6}$ protein was successfully expressed in E. coli BL21 (DE3). This provides a valuable source of porcine MID1IP1 for further experimentation into MIDIIP1 function for further investigation of meat quality in swine.

\section{ACKNOWLEDGMENTS}

Research supported by the Project of Recommended International Advanced Agricultural Science and Technology Plan, Ministry of Agriculture of China (\#2011-G35).

\section{REFERENCES}

Aipoalani DL, O'Callaghan BL, Mashek DG, Mariash CN, et al. (2010). Overlapping roles of the glucose-responsive genes, S14 and S14R, in hepatic lipogenesis. Endocrinology 151: 2071-2077.

Berti C, Fontanella B, Ferrentino R and Meroni G (2004). Mig12, a novel Opitz syndrome gene product partner, is expressed in the embryonic ventral midline and co-operates with Mid1 to bundle and stabilize microtubules. $B M C$ Cell Biol. 5: 9.

Brown SB, Maloney M and Kinlaw WB (1997). Spot 14" protein functions at the pretranslational level in the regulation of hepatic metabolism by thyroid hormone and glucose. J. Biol. Chem. 272: 2163-2166.

Colbert CL, Kim CW, Moon YA, Henry L, et al. (2010). Crystal structure of Spot 14, a modulator of fatty acid synthesis. Proc. Natl. Acad. Sci. U. S. A. 107: 18820-18825.

Freake HC and Oppenheimer JH (1987). Stimulation of S14 mRNA and lipogenesis in brown fat by hypothyroidism, cold exposure, and cafeteria feeding: evidence supporting a general role for S14 in lipogenesis and lipogenesis in the maintenance of thermogenesis. Proc. Natl. Acad. Sci. U. S. A. 84: 3070-3074.

Grillasca JP, Gastaldi M, Khiri H, Dace A, et al. (1997). Cloning and initial characterization of human and mouse Spot 14 genes. FEBS Lett. 401: 38-42.

Inoue J, Yamasaki K, Ikeuchi E, Satoh S, et al. (2011). Identification of MIG12 as a mediator for stimulation of lipogenesis by LXR activation. Mol. Endocrinol. 25: 995-1005. 
Jump DB, Narayan P, Towle H and Oppenheimer JH (1984). Rapid effects of triiodothyronine on hepatic gene expression. Hybridization analysis of tissue-specific triiodothyronine regulation of mRNAS14. J. Biol. Chem. 259: 2789-2797.

Jump DB and Oppenheimer JH (1985). High basal expression and 3,5,3'-triiodothyronine regulation of messenger ribonucleic acid S14 in lipogenic tissues. Endocrinology 117: 2259-2266.

Kim CW, Moon YA, Park SW, Cheng D, et al. (2010). Induced polymerization of mammalian acetyl-CoA carboxylase by MIG12 provides a tertiary level of regulation of fatty acid synthesis. Proc. Natl. Acad. Sci. U. S. A. 107: 9626-9631.

Kinlaw WB, Church JL, Harmon J and Mariash CN (1995). Direct evidence for a role of the "spot 14" protein in the regulation of lipid synthesis. J. Biol. Chem. 270: 16615-16618.

Liaw CW and Towle HC (1984). Characterization of a thyroid hormone-responsive gene from rat. J. Biol. Chem. 259: 7253-7260.

Seelig S, Liaw C, Towle HC and Oppenheimer JH (1981). Thyroid hormone attenuates and augments hepatic gene expression at a pretranslational level. Proc. Natl. Acad. Sci. U. S. A. 78: 4733-4737.

Tsatsos NG, Augustin LB, Anderson GW, Towle HC, et al. (2008). Hepatic expression of the SPOT 14 (S14) paralog S14related (Mid1 interacting protein) is regulated by dietary carbohydrate. Endocrinology 149: 5155-5161.

Wang C M, Guo Y J, Li H J, Han L Q, et al. (2012). Cloning and prokaryotic expression of sus scrofa thyroid hormone responsive SPOT14 gene (THRSP) and affinity purification of its fused protein. J. Agric. Biotechnol. 20: 301-307.

Zhu Q, Mariash A, Margosian MR, Gopinath S, et al. (2001). Spot 14 gene deletion increases hepatic de novo lipogenesis. Endocrinology 142: 4363-4370.

Zhu Q, Anderson GW, Mucha GT, Parks EJ, et al. (2005). The Spot 14 protein is required for de novo lipid synthesis in the lactating mammary gland. Endocrinology 146: 3343-3350. 